\title{
Nonexponential statistics of fluorescence photobleaching
}

\author{
Andrew J. Berglund ${ }^{\text {a) }}$ \\ Norman Bridge Laboratory of Physics 12-33, California Institute of Technology, Pasadena, California 91125
}

(Received 26 February 2004; accepted 25 May 2004)

\begin{abstract}
In this paper, I consider theoretical models of the decay via photobleaching of a sample of surface-immobilized fluorescent molecules excited by a spatially varying laser intensity profile. I show that, with mild restrictions on the photobleaching mechanism, the fluorescence decay measured in a nonuniform excitation profile is always nonexponential. Under the same conditions, the fluorescence decay can always be approximated by a discrete sum of exponentials. A particular example is given in which a homogeneous population of fluorophores with a single (intensity-dependent) photobleaching lifetime, when illuminated by a Gaussian laser, exhibits power law fluorescence decay at long times. These results indicate that the observation of multiple exponentials in single molecule or ensemble photobleaching lifetime measurements can arise solely as an artifact of a spatially varying laser profile and is not necessarily indicative of heterogeneity in molecular internal states, conformation, or local environment. (C) 2004 American Institute of Physics. [DOI: 10.1063/1.1773162]
\end{abstract}

\section{INTRODUCTION}

Fluorescent dye molecules and site-specific labelling techniques are now routinely used for probing structure and dynamics at the scale of individual biological molecules. ${ }^{1}$ However, dye molecules exhibit complex photodynamics including irreversible "photobleaching" often in the millisecond-to-second time range that complicate the interpretation of experimental data. ${ }^{1,2}$ Understanding photobleaching lifetimes is therefore a problem of fundamental importance for the field of single-molecule fluorescence microscopy. Correspondingly, experimental and theoretical studies of photobleaching of single dye molecules have received much attention in the literature. ${ }^{2-11}$

The seemingly ubiquitous appearance of multiple exponential distributions in measurements of ensemble and single-molecule fluorescence photobleaching lifetimes ${ }^{2-5,8,10}$ indicates the difficulty in interpreting such results. These multiple-exponential lifetime distributions often arise as a result of heterogeneity in the molecular population or as a result of a spatially varying laser intensity profile (or a combination of both). In this paper, I consider some mathematical properties of the latter mechanism. An analytical characterization of the class of completely monotonic functions will be used to derive sufficient conditions for nonexponential statistics in measurements of fluorescence decay from surface immobilized samples using confocal epifluorescence microscopy. The relevance of completely monotonic functions has also been recognized in polymer physics ${ }^{12,13}$ and network analysis. ${ }^{14}$ Here, I show that a broad class of models that exhibits a driving intensity-dependent photobleaching rate gives rise to nonexponential decay but exhibits fictitious exponential "lifetimes" whenever the sample is illuminated by a Gaussian laser profile. These results indicate that care must be taken in interpreting the appearance of multiple ex-

${ }^{\text {a)} E l e c t r o n i c ~ m a i l: ~ b e r g l u n d @ ~ c a l t e c h . e d u ~}$ ponential distributions in numerical fits to measured fluorescence decay data. In particular, without restrictions on the laser excitation profile across the observation area, the presence of multiple exponential lifetimes in a fluorescence decay curve is not sufficient for making a determination about the heterogeneity of the molecular population. The paper is organized as follows: Section II contains mathematical results, which characterize a class of models giving rise to nonexponential statistics. These general results are then applied to an example calculation in Sec. III, and some conclusions and possible extensions are presented in Sec. IV.

\section{SUFFICIENT CONDITIONS FOR NONEXPONENTIAL DECAY}

In this section, I will borrow some ideas from classical functional analysis and, in particular, I will introduce completely monotonic $(\mathrm{CM})$ functions and their characterization in terms of Laplace transforms. CM functions play an important role in analyzing nonexponential kinetics, and it will be seen that CM functions can always be approximated by discrete sums of exponentials. Here, a curve is considered "nonexponential" if it cannot be written as a single exponential, and "multiexponential" if it can be written as a convex sum of at least two exponentials with different time constants. Results concerning CM functions and their relation to Laplace transforms are taken from Widder's book. ${ }^{15} \mathrm{~A}$ function $f(t)$ is said to be increasingly (decreasingly) monotonic on the interval $[0, \infty)$ if $f\left(t_{2}\right)>f\left(t_{1}\right)\left[f\left(t_{2}\right)<f\left(t_{1}\right)\right]$ for all $0 \leqslant t_{1}<t_{2}<\infty$. A function $f(t)$ is said to be completely monotonic on the interval $[0, \infty)$ if it is infinitely differentiable and the following inequality holds for all $t>0$,

$$
(-1)^{n} \frac{d^{n}}{d t^{n}} f(t) \geqslant 0, \quad n=\{0,1,2 \ldots\} .
$$

That is, CM functions have derivatives of alternating sign for all $t>0$. The point $t=0$ may be included in the domain of 
(complete) monotonicity by requiring $f(t)$ to be right continuous at $t=0: f(0)=f\left(0^{+}\right)$. In what follows, the domain of monotonicity is always understood to be $0 \leqslant t<\infty$. CM functions are positive, nonincreasing, convex up, etc. The central analytical result which characterizes CM functions for our purposes is Bernstein's theorem (see Ref. 15), which states that $f(t)$ is $\mathrm{CM}$ if and only if

$$
f(t)=\int_{0}^{\infty} e^{-y t} d \widetilde{F}(y),
$$

where the integral converges and $\widetilde{F}(y)$ is bounded and nondecreasing [i.e., $\widetilde{F}(y)$ is a measure on $[0, \infty)]$. It follows immediately that a sufficient condition for $f(t)$ to be $\mathrm{CM}$ is the representation

$$
f(t)=\int_{0}^{\infty} e^{-y t} \tilde{f}(y) d y,
$$

where the integral converges and $\widetilde{f}(y)$ is non-negative, which can be seen by setting $\widetilde{F}(y)=\int_{0}^{y} \widetilde{f}\left(y^{\prime}\right) d y^{\prime}$ and using Bernstein's theorem. The Laplace representation of Bernstein's theorem shows intuitively that CM functions are the limit of a convex combination of exponentials with time constants $1 / y$ and weights $d \widetilde{F}(y)$. One can show that exponentials, stretched exponentials $\left[\exp \left(-t^{a}\right)\right.$ with $\left.0<a<1\right]$, and power laws $\left(t^{-b}\right.$ with $\left.b>0\right)$ are completely monotonic, and also that convex combinations, products, integrals, and derivatives of completely monotonic functions are themselves completely monotonic (up to signs and additive constants). Thus, while the characterizations (1) and (2) may seem restrictive, CM functions represent a quite general class of "decaying" curves.

Feldmann and Whitt ${ }^{14}$ state that any CM function $f(t)$ can be approximated by a discrete sum of exponentials:

$$
f(t)=\sum_{k=0}^{\infty} c_{k} e^{-\nu_{k} t}
$$

and they give an efficient algorithm for finding the (nonnegative) parameters $\nu_{k}$ and $c_{k}$. An example given in Sec. III shows that the expansion (4) can converge surprisingly fast, so that often only a few terms in the expansion are necessary for an excellent approximation.

Having seen that CM functions are intimately connected with both continuous and discrete distributions of exponentials, let us now consider a generalized fluorescence photobleaching experiment and attempt to use the properties of $\mathrm{CM}$ functions to find conditions on the photobleaching mechanism and the laser intensity profile that guarantee nonexponential fluorescence decay. Consider a measurement typical of confocal microscopy in an epifluorescence configuration, in which dye molecules are uniformly distributed on a two-dimensional surface and excited by a laser with a cylindrically symmetric intensity profile. Let $u(r) \geqslant 0$ be the average fluorescence intensity from a molecule at a distance $r$ from the beam centroid normalized so that $u(0)=1$. The fluorescence intensity $u(r)$ and the laser excitation profile are assumed to be smoothly and monotonically (but not necessarily completely monotonically) related so that we do not need to write the laser intensity profile explicitly. This is a very general assumption, which simply states that fluorescence increases smoothly with increasing laser power. Suppose also that the instantaneous rate at which a molecule photobleaches is given by $\gamma[u(r)] \geqslant 0$. An intensity dependent rate is a good model for the photodestruction of dye molecules which are sufficiently photostable that photobleaching lifetimes $\left(10^{-2}-10^{-1} \mathrm{~s}\right)$ are slow compared to other photodynamics such as fluorescence "blinking" $\left(10^{-6}-10^{-3} \mathrm{~s}\right)$ and electronic transitions $\left.\sim 10^{-9} \mathrm{~s}\right){ }^{9}$ For this separation of time scales, a molecule under fixed excitation has a constant, steady-state probability of photobleaching in any time interval.

With these definitions, the total time-dependent (average) fluorescence signal $\sigma(t)$ is given by an integral over the entire surface

$$
\sigma(t)=\int_{0}^{\infty} 2 \pi r u(r) e^{-\gamma[u(r)] t} d r .
$$

Note that $\sigma(t)$ represents the average fluorescence decay from a population of identical molecules whose fluorescence and photobleaching properties are characterized by the functions $u(r)$ and $\gamma[u(r)] . \sigma(t)$ may also represent the probability of a particular photobleaching lifetime measured in single molecule measurements, so that these arguments are not limited to ensemble experiments. It is clear that all functions $\sigma(t)$ for which the integral (5) converges (i.e., the total fluorescence signal is finite) are CM, since

$$
\begin{aligned}
(-1)^{n} \frac{d^{n}}{d t^{n}} \sigma(t) & =\int_{0}^{\infty} 2 \pi r \gamma[u(r)]^{n} u(r) e^{-\gamma[u(r)] t} d r \\
& \geqslant 0
\end{aligned}
$$

and the integrand in the above inequality is nonnegative for all $n$. Therefore, we arrive at the first analytical result of this paper.

Result 1. For a surface-immobilized molecular distribution, every photobleaching mechanism that is described by an intensity-dependent rate gives rise to completely monotonic fluorescence decay.

This result holds even for discontinuous functions $u$ and $\gamma$.

I will now impose some restrictions on the boundedness, smoothness, and monotonicity of $u(r)$ and $\gamma[u(r)]$. Let

$$
\left.u^{\prime}(\rho) \equiv \frac{\partial u(r)}{\partial r}\right|_{r=\rho}
$$

and

$$
\left.\gamma^{\prime}[v] \equiv \frac{\partial \gamma[u(r)]}{\partial u(r)}\right|_{u(r)=v} .
$$

First, it is assumed that $u(r)$ is continuous, differentiable, and decreases monotonically to 0 on the interval $0<r<\infty$. Similarly, it is assumed that the photobleaching rate $\gamma[u(r)]$ is continuous, differentiable (with respect to $u(r)$ ), bounded, and increases monotonically in the interval $0<u(r)<1$ with $\gamma[0]=0$. These are very general requirements that simply state that the laser profile is smooth and localized, and molecules that fluoresce more brightly also photobleach more quickly (with a suitably smooth map between these rates). 
Finally, it is assumed that the spatial extent of the excitation profile is much smaller than the experimental field-of-view so that we need not distinguish between fluorescence and observed fluorescence. This is a typical situation in confocal epifluorescence microscopy. For brevity, if $u(r)$ and $\gamma[u(r)]$ each satisfy all of these restrictions, I will say that they are smooth and spatially varying (SSV). SSV conditions will typically arise in diffraction limited, high-resolution confocal microscopy conditions, unless special precautions are taken to restrict the field-of-view. Total internal reflection spectroscopy, in which an evanescent wave field illuminates a surface distribution over a large area, is a notable situation in which SSV conditions do not apply.

Equation (5) gives a convenient integral decomposition of the decay curve $\sigma(t)$. If they are SSV, the monotonicity of $u(r)$ and $\gamma[u(r)]$ guarantees the existence of their inverse functions $u^{-1}$ and $\gamma^{-1}$ so that letting $y=\gamma[u(r)]$ and using the chain rule, we have

$$
r d r=\frac{u^{-1}\left[\gamma^{-1}(y)\right]}{\gamma^{\prime}\left[\gamma^{-1}(y)\right] u^{\prime}\left\{u^{-1}\left[\gamma^{-1}(y)\right]\right\}} d y .
$$

The change of variables (7) then permits us to rewrite $\sigma(t)$ in the form of Eq. (3)

$$
\sigma(t)=\int_{0}^{\infty} e^{-y t} \widetilde{\sigma}(y) d y,
$$

where the frequency-weighting function is given by

$$
\widetilde{\sigma}(y)=\frac{-2 \pi \gamma^{-1}(y) u^{-1}\left[\gamma^{-1}(y)\right]}{\gamma^{\prime}\left[\gamma^{-1}(y)\right] u^{\prime}\left\{u^{-1}\left[\gamma^{-1}(y)\right]\right\}}, \quad 0 \leqslant y \leqslant y_{0}
$$

and $\widetilde{\sigma}(y)=0$ elsewhere, with

$$
y_{0}=\gamma[u(r)=1] .
$$

When it exists, Eq. (9) is the exact expression for the inverse Laplace transform of Eq. (5) and by Bernstein's theorem, $\widetilde{\sigma}(y)$ completely characterizes the CM decay curve $\sigma(t)$.

Here, I will note a few properties of $\widetilde{\sigma}(y)$ that arise from the SSV conditions imposed on $\gamma$ and $u$. We see by inspection that $\widetilde{\sigma}(y) \geqslant 0$ for all $y \geqslant 0$, as required by complete monotonicity (recall that $\gamma$ is monotonically increasing while $u$ is monotonically decreasing, so that their derivatives are of opposite sign). The numerator in (9) is never zero for $0<y$ $<y_{0}$, which can be seen by letting $y=\gamma[u(r)]$ and considering $0<r<\infty$. Furthermore, we see that for SSV conditions, the denominator is finite since $\gamma[u(r)]$ and $u(r)$ are differentiable. Therefore, $\widetilde{\sigma}(y)$ given by Eq. (9) is everywhere nonzero in $0<y<y_{0}$.

Now, consider the case that $\sigma(t)$ is exponential. Since the (unique) inverse Laplace transform of an exponential is a delta function $\widetilde{\sigma}(y) \propto \delta\left(y-y_{\delta}\right)$, which is everywhere zero except at an isolated point of discontinuity $y=y_{\delta}$, we see that if $\widetilde{\sigma}(y)$ given by Eq. (9) is SSV (i.e., nonzero in $0<y$ $\left.<y_{0}\right)$ then $\sigma(t)$ cannot be exponential, nor can it be given by any finite sum of exponentials. Therefore, we arrive at the second analytical result of this work.

Result 2. For "smooth and spatially varying" conditions, the fluorescence decay measured from a surface-immobilized distribution of molecules is always nonexponential.
Leaving aside the mathematical details, the main point is that, under SSV conditions of smoothness and monotonicity, $u(r)$ and $\gamma[u(r)]$ cannot conspire to make $\widetilde{\sigma}(y)$ given by Eq. (9) into a delta function, and thus the decay is never exponential.

All models $\gamma[u(r)]$ that give photobleaching lifetimes with a nontrivial, analytic dependence on intensity are SSV, which includes all models I am aware of in the literature. In particular, the widely accepted three-level and five-level triplet-mediated photobleaching mechanisms (5), (8), and (9) are examples of SSV models. Furthermore, many experiments are performed with a Gaussian laser profile, so that at large $r, u(r)$ decays as $\exp \left(-k r^{2}\right)$ with $k \geqslant 0$. This statement relies on the fact that saturation effects become negligible in the low-intensity limit, so that the excitation and emission rates are proportional at sufficiently large $r$. It also holds even for multiple-photon excitation, since products of Gaussians are still Gaussian. It is clear therefore that $u(r)$ derived from a Gaussian excitation profile is SSV. As a particular example of Result 2, I conclude that for measurements on uniform surface-immobilized samples under a very general class of photobleaching mechanisms, when precautions are not taken to restrict the excitation profile, every fluorescence photobleaching decay curve measured with a Gaussian laser exhibits nonexponential decay. I emphasize that the nonexponential character arises solely from the variation in excitation intensity, in which short-time statistics are dominated by brightly illuminated molecules and late-time statistics are dominated by long-lived molecules at the outskirts of the intensity profile. An extension of the present results to the case of a spatially varying collection efficiency $\eta(r) \leqslant 1$ or a nonuniform distribution of molecules should be straightforward.

\section{EXAMPLE: LINEAR PHOTOBLEACHING RESPONSE}

In this section, I apply the general results of Sec. II to the linear photobeaching model $\gamma[u(r)]=\alpha u(r)$ with a saturating two-level emitter model of fluorescence. The fluorescence exhibits power-law decay, but the resulting curve is well-approximated by the sum of only three or four exponentials over four orders of magnitude in time. This example is meant to illustrate the appearance of multiple fictitious lifetimes in a measurement performed on a homogeneous population with a single photobleaching parameter $\alpha$.

Dyes at position $r$ are excited from the ground to the first excited electronic state at a rate $\Gamma_{e} I(r)$, where $I(r)$ is the laser intensity in suitable units with $I(0)=1$, and relax by fluorescence photon emission at the rate $\Gamma_{r}$. In the excited state, the molecule has a constant probability of photobleaching in any constant time interval. Molecules at position $r$ fluoresce at a (normalized) rate $u(r)$ given by

$$
u(r)=\frac{(1+\kappa) I(r)}{1+\kappa I(r)},
$$

where $\kappa=\Gamma_{e} / \Gamma_{r}$ parametrizes the maximum laser intensity. This model is equivalent to an exponential distribution of photobleaching lifetimes (at constant laser intensity) and also 

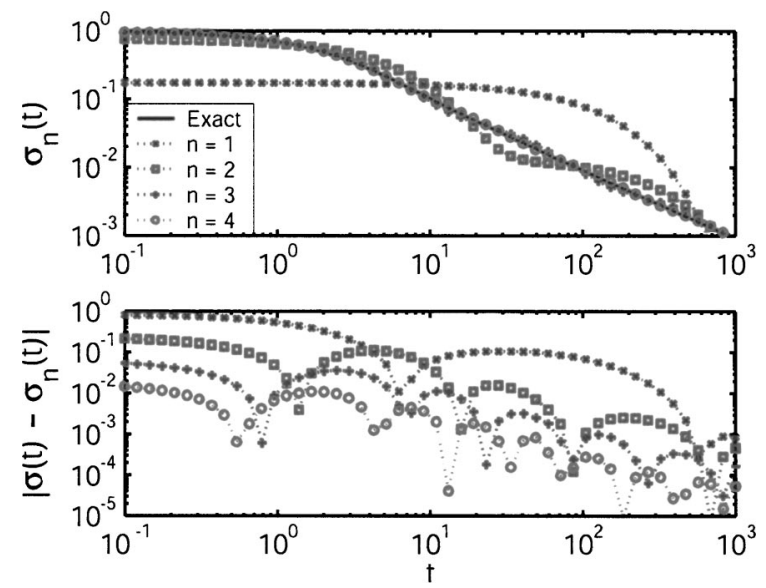

FIG. 1. A log-log plot of the ratio of a multiexponential fit $\sigma_{n}(t)$ to the exact form of $\sigma(t)$, with $\gamma_{0}=1$ and $\kappa=2$. These parameters are typical of dye molecules driven into the saturation regime.

equivalent to an exponential distribution of "death numbers," the number of photons emitted before photodestruction. The parameter $\alpha$ determines the average lifetime $[1 / \alpha u(r)]$ and average death number $\left(\Gamma_{r} / \alpha\right)$ in these equivalent models.

The average fluorescence intensity $\sigma(t)$ collected over the entire surface is given by

$$
\sigma(t)=\int_{0}^{\infty} u(r) \exp [-\alpha u(r) t] 2 \pi r d r .
$$

For a Gaussian beam profile $I(r)$ with waist $w$, we may substitute into Eq. (9) to find the frequency decomposition

$$
\widetilde{\sigma}(y)=\left\{\begin{array}{l}
\frac{n_{o}}{1-y / \gamma_{o}}, \quad y \leqslant \alpha \\
0, \quad y>\alpha
\end{array}\right.
$$

with

$$
n_{o}=\frac{\pi w^{2}}{2 \alpha}
$$

and

$$
\gamma_{o}=\alpha(1+1 / \kappa) .
$$

Taking the Laplace transform of Eq. (13), we find

$$
\sigma(t)=n_{o} \gamma_{0} e^{-\gamma_{0} t}\left[\operatorname{Ei}\left(\gamma_{0} t\right)-\operatorname{Ei}\left(\frac{\gamma_{0} t}{1+\kappa}\right)\right],
$$

where Ei is the exponential integral function. ${ }^{16} \sigma(t)$ given by Eq. (15) is a nonexponential distribution which decays as $1 / t$ at long times. From Eq. (13) it is clear that there is a natural fastest timescale $1 / \alpha$ to the decay, but that as $y$ approaches 0 , $\widetilde{\sigma}(y)$ approaches a constant value so that exponentials with arbitrarily long lifetimes are all represented with nonzero weight.

Figure 1 shows a log-log plot of the ratio of a multiexponential fit $\sigma_{n}(t)$ to the exact form of $\sigma(t)$, with $\gamma_{0}=1$ and $k=2$. These parameters are typical of dye molecules driven into the saturation regime. Letting

$$
\sigma_{n}(t)=c_{0}+\sum_{j=1}^{n} c_{j} e^{-v_{j} t}
$$

and fitting the parameters $\left\{c_{j}, \nu_{j}\right\}$ using a nonlinear leastsquares fitting routine, we see that, over these four orders of magnitude in time, the fit becomes quite good for $n=3,4$. The relationship between the $n$ exponential lifetimes, which arise from fitting the exact result, and the two free parameters of the model $\alpha$ and $\kappa$ is not at all obvious and depends strongly on the time-interval of the fit. In a realistic experimental scenario, the presence of a finite background signal and photon counting statistics may easily dominate the residual fitting error for $n$ larger than about 2 or 3 . Thus, the number of exponential lifetimes observed in a sample that exhibits completely monotonic, nonexponential decay can vary solely based on signal-to-noise and fluorescence detection efficiency with no variation in molecular internal states or local environments.

\section{CONCLUSIONS}

In this paper, I have used analytical results from the theory of completely monotonic functions to show the existence of nonexponential decay in fluorescence photobleaching measurements in a spatially varying excitation profile. Under these conditions, I have shown that fluorescence decay curves that arise under models exhibiting an analytic intensity-dependent photobleaching rate are nonexponential and are furthermore well-approximated by a discrete sum of exponentials. The appearance and utility of multiple exponential fits to experimental data is therefore not sufficient for determining the heterogeneity of a molecular population unless the laser excitation profile has been restricted so that there is essentially no variation of the laser intensity over the field of view. The results presented here are strictly applicable only to measurements performed on surfaceimmobilized samples, but it seems unlikely to the author that additional complexity due to, for example, free diffusion in solution or concentration-enhanced photobleaching will conspire to mask the essentially nonexponential character of fluorescence photobleaching in a nonuniform excitation profile. However, these questions remain to be analyzed in future work.

\section{ACKNOWLEDGMENTS}

This work was performed in Hideo Mabuchi's group, and the author gratefully acknowledges their support and technical advice. The author also thanks Kevin McHale for a careful reading of the manuscript. This work was supported by the Institute for Collaborative Biotechnologies through grant DAAD19-03-D-0004 from the U.S. ARO, and by the NSF under Grant Nos. EIA-0323542 and EIA-0113443. Any opinions, findings, and conclusions or recommendations expressed in this material were those of the author and do not necessarily reflect the views of either funding agency.

\footnotetext{
${ }^{1}$ S. Weiss, Science 283, 1676 (1999).

${ }^{2}$ X. S. Xie and J. K. Trautman, Annu. Rev. Phys. Chem. 49, 441 (1998).

${ }^{3}$ J. Widengren and R. Rigler, Bioimaging 4, 149 (1996).
} 
${ }^{4}$ C. Eggeling, L. Brand, and C. A. M. Seidel, Bioimaging 5, 105 (1997).

${ }^{5}$ C. Eggeling, J. Widengren, R. Rigler, and C. A. M. Seidel, Anal. Chem. 70, 2651 (1998).

${ }^{6}$ E. J. Sanchez, L. Novotny, G. R. Holtom, and X. S. Xie, J. Phys. Chem. A 101, 7019 (1997).

${ }^{7}$ L. Ying and X. S. Xie, J. Phys. Chem. B 102, 10399 (1998).

${ }^{8}$ S. Wennmalm and R. Rigler, J. Phys. Chem. B 103, 2516 (1999).

${ }^{9}$ A. Molski, J. Chem. Phys. 114, 1142 (2001).

${ }^{10}$ D.-S. Ko, J. Chem. Phys. 120, 2530 (2004).
${ }^{11}$ R. A. Mathies, K. Peck, and L. Stryer, Anal. Chem. 62, 1786 (1990).

${ }^{12}$ G. del Piero and L. Deseri, Q. Appl. Math. LIII, 273 (1995).

${ }^{13}$ R. S. Anderssen and R. J. Loy, J. Rheol. 46, 1459 (2002).

${ }^{14}$ A. Feldmann and W. Whitt, Perform. Eval. 31, 245 (1998).

${ }^{15}$ D. V. Widder, The Laplace Transform (Princeton University Press, Princeton, NJ, 1946).

${ }^{16}$ Handbook of Mathematical Functions, With Formulas, Graphs, and Mathematical Tables, 9th ed., edited by M. Abramowitz and I. Stegun (Dover, New York, 1972). 